\title{
Resolution of calcinosis using bisphosphonates in overlap syndrome - a case report
}

\author{
Mitchell Platter ${ }^{*}$ D, Brian Pugmire and Reshma Patel
}

\begin{abstract}
Introduction: Calcinosis cutis is a common complication of pediatric rheumatologic diseases. However, there is currently no consensus on first-line treatment. Bisphosphonates have been described as a successful treatment in several case studies, but most of these cases are limited to patients with isolated juvenile dermatomyositis or systemic sclerosis. Specifically, there are limited reports of their usefulness in treating overlap syndromes and mixed connective tissue disorders.

Case presentation: We describe the case of a 13 year-old girl with overlap syndrome with features of juvenile dermatomyositis and systemic lupus erythematosus. After 22 months of extensive immunosuppressive therapy, including monthly IVIG and Rituximab, she continued to have pain and weakness of the lower extremities. A CT scan was performed which showed significant multifocal soft tissue calcifications of the pelvis. She was started on treatment with oral alendronate with the goal of improving her calcinosis and improving her symptoms. After several months of therapy, our patient reported subjective improvement of her lower extremity pain and weakness, as well as complete resolution of abnormalities previously seen on physical examination. A repeat CT scan of the pelvis was performed after 11 months of therapy and demonstrated complete resolution of the previously seen calcinosis.

Conclusions: We report the successful treatment of soft tissue calcinosis with oral bisphosphonates in a patient with juvenile dermatomyositis-systemic lupus erythematosus overlap syndrome. These results provide further evidence that bisphosphonates can be used successfully to treat calcinosis cutis in pediatric rheumatologic disorders. Additionally, the results provide new evidence that they can be used specifically in juvenile dermatomyositis-systemic lupus erythematosus overlap syndrome, which has not been previously reported.
\end{abstract}

Keywords: Overlap syndrome, Juvenile dermatomyositis, Systemic lupus erythematosus, Calcinosis, Bisphosphonate, Alendronate, Case report

\section{Background}

Pediatric autoimmune connective tissue disorders (ACTD) encompass a wide range of diagnoses, each with their own unique symptoms and complications. One of the most common sources of morbidity amongst these diseases is subcutaneous calcinosis, which has been

\footnotetext{
*Correspondence: mplatter2@valleychildrens.org

Department of Pediatric Rheumatology at Valley Children's Hospital, 9300 Valley Children's Place, Madera, CA 93720, USA
}

identified in $20-40 \%$ of cases of juvenile dermatomyositis (JDM) [1-3]. Calcinosis is most commonly identified in JDM and systemic sclerosis, but it can be seen in nearly all ACTDs [4]. The calcinosis in these disorders most commonly affects the knee joints, elbow joints, and hip processes [5]. Although it is a well-recognized source of morbidity in these disorders, there are currently no standardized recommendations for its treatment [3]. Of the ACTDs, treatment of calcinosis in JDM and systemic sclerosis has been most frequently reported, although

(C) The Author(s). 2021 Open Access This article is licensed under a Creative Commons Attribution 4.0 International License, which permits use, sharing, adaptation, distribution and reproduction in any medium or format, as long as you give appropriate credit to the original author(s) and the source, provide a link to the Creative Commons licence, and indicate if changes were made. The images or other third party material in this article are included in the article's Creative Commons licence, unless indicated otherwise in a credit line to the material. If material is not included in the article's Creative Commons licence and your intended use is not permitted by statutory regulation or exceeds the permitted use, you will need to obtain permission directly from the copyright holder. To view a copy of this licence, visit http://creativecommons.org/licenses/by/4.0/ The Creative Commons Public Domain Dedication waiver (http://creativecommons.org/publicdomain/zero/1.0/) applies to the data made available in this article, unless otherwise stated in a credit line to the data. 
there are currently no large scale, case-controlled studies. Rheumatologists often treat calcinosis with immunomodulating agents such as intravenous immunoglobulin (IVIG) and systemic glucocorticoids, or alternative agents such as bisphosphonates and calcium channel blockers [6]. Of these choices, bisphosphonates have been inconsistently associated with complete resolution of calcinosis in various case reports of patients with JDM $[7,8]$. They are also noted to be relatively safe, with short-term side effects consisting of bone pain, hypocalcemia, and gastrointestinal symptoms such as diarrhea and abdominal pain. Longer-term use has been infrequently associated with impaired mineralization and nephrocalcinosis [9]. Although demonstrated to be effective and safe in treating calcinosis in JDM and systemic sclerosis, there is very limited data on the use of bisphosphonates in other rheumatologic disorders such as overlap syndromes and mixed connective tissue disorders (MCTD). In this review, we report the case of a 13 year-old girl with JDM-systemic lupus erythematosus (SLE) overlap syndrome who developed large, bulky calcinosis of the pelvis while on immunosuppressive therapy and demonstrated complete resolution after treatment with oral bisphosphonate therapy.

\section{Case presentation}

We report the case of a 13 year-old, Hispanic girl who was diagnosed with JDM-SLE overlap syndrome at age 10. She initially presented to our hospital with complaints of symmetric upper and lower extremity muscle weakness and a photosensitive rash for 1 week prior to presentation. On physical examination, she had 3/5 strength in the proximal muscles of the bilateral upper and lower extremities, with an initial CMAS score of 6 . Her cutaneous manifestations included a malar rash, heliotrope rash, and violaceous plaques on the dorsal aspect of the metacarpophalangeal joints consistent with Gottron papules. Her initial laboratory examination was significant for elevated muscle enzymes with an AST of 611, ALT of 392, alkaline phosphatase of 118, LDH of 1740 , and creatine kinase of 16,012 . She subsequently had a lower extremity MRI performed that showed diffuse, symmetric intramuscular T2 hyper intense signal within the gluteal adductor, extensor, and flexor musculature, consistent with diffuse proximal lower extremity myositis. On further laboratory examination, she was found to have a strongly positive ANA of 1:1280 with speckled pattern and a positive dsDNA by Crithidia of 19 (strongly positive $>9$ ), with normal complements and negative Smith/RNP antibodies. With her clinical findings, laboratory evaluation, and radiologic findings she was diagnosed with a likely overlap syndrome with mostly features of JDM and SLE. As an inpatient, she was started on "protocol A" based on the "three consensus protocols for the initial treatment of moderate to severe juvenile dermatomyositis" [10]. She received IV methylprednisolone $30 \mathrm{mg} / \mathrm{kg} /$ day for 3 days and was started on subcutaneous methotrexate $15 \mathrm{mg} / \mathrm{m}^{\wedge} 2$ weekly. She was also started on oral hydroxychloroquine $200 \mathrm{mg} /$ day, and after 3 days of methylprednisolone she was transitioned to oral prednisone $2 \mathrm{mg} / \mathrm{kg} /$ day divided BID. After the high dose steroids, her CK decreased to 5756 and her muscle strength improved as per PT evaluation, and she was discharged home 1 week after admission.

As an outpatient, she was scheduled to receive aggressive physical therapy. However, shortly after being discharged home she had two episodes of falling due to weakness and was readmitted 2 weeks after her initial presentation. She was found to have worsening proximal muscle strength at $2 / 5$ in the lower extremities, a return of her photosensitive rash, and an increase in her CK to 8784. At this point she was switched to "Protocol B" of the above mentioned treatment recommendations and was given IVIG 2G/kg [10]. Given the severity of her weakness and her typical photosensitive rash, she was given another course of IV methylprednisolone $30 \mathrm{mg} /$ $\mathrm{kg} /$ day for 3 days. She was then transferred to the inpatient rehabilitation unit where she completed 1 week of aggressive daily physical and occupational therapy. She was maintained on monthly IVIG and subcutaneous methotrexate along with a daily oral tapered prednisone regimen. She had another flare 11 months after her initial diagnosis which presented as a new inflammatory synovitis in the knee and wrist. She also had return of her malar rash, a worsening rash over her bilateral shoulders and chest (shawl sign), and elevation of her CK to 12,461 , likely secondary to noncompliance with her outpatient IVIG infusions. She again received IV methylprednisolone $30 \mathrm{mg} / \mathrm{kg} /$ day for 3 days and IVIG $2 \mathrm{G} / \mathrm{kg}$. After discharge, despite better compliance with her monthly IVIG infusions, she continued to have episodes of weakness and elevated muscle enzymes with difficulty weaning off of prednisone. At 16 months after initial diagnosis, she was given two doses of Rituximab $750 \mathrm{mg} / \mathrm{m}^{\wedge} 2$ intravenous infusions for recalcitrant disease, and was maintained on IVIG $70 \mathrm{~g}$ every month. Two months after completion of her Rituximab course there was drastic improvement and eventually normalization of her serological muscle enzymes.

At 22 months after diagnosis, she continued to complain of pain in her lower extremities despite receiving monthly IVIG and intense ongoing physical therapy. During an outpatient physical examination at this time, she was very hesitant to sit on the floor, and had to use Gower's maneuver to stand from sitting. There was also new, decreased passive range of motion of the right hip when compared to the left. Estimated CMAS score at 


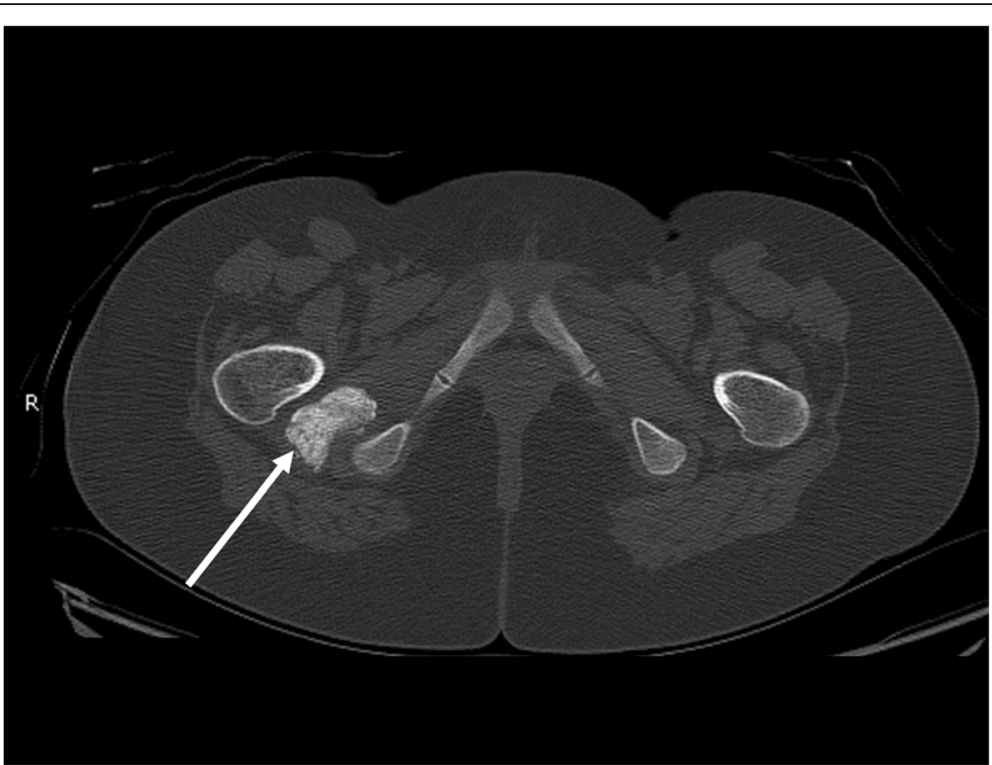

Fig. 1 Axial CT image of the pelvis demonstrates a large area of bulky soft tissue calcification in the right ischiofemoral space (white arrow)

this time was improved to 47. Her muscle enzymes were also now persistently normal, so it was unclear if this was anatomical versus isolated muscle group weakness. Due to her continued symptoms and asymmetrical findings, an x-ray of the pelvis was ordered, which showed a rounded ossification near the right femoral neck, a smaller ossification near the left medial acetabulum, and multiple muscular calcifications in the adductor groups near the hip. A CT scan of the pelvis was ordered to further evaluate these findings, which showed a region of bulky, dense, soft tissue calcification measuring $3.8 \times 2.3$ $\mathrm{cm}$ within the ischiofemoral region on the right (Figs. 1 and 2), as well as scattered coarse linear calcifications within the abductor muscles bilaterally (Fig. 3). It also showed a $5.0 \times 1.0 \mathrm{~cm}$ ovoid calcification in the left obturator internus muscle near the ischium. It was felt that these calcifications were contributing to her limitations in range of motion and subjective complaints, so additional therapy was pursued. She was started on the bisphosphonate alendronate orally at $10 \mathrm{mg} /$ day in addition to her ongoing therapy with hydroxychloroquine, weekly methotrexate, and monthly IVIG.

Our patient continued to follow up every 3 months in our clinic. Three months after starting alendronate she reported some improvement in her pain and strength, but on physical examination still had difficulty with squatting, and still used Gower's maneuver when standing. A repeat $\mathrm{x}$-ray was ordered at 7 months on bisphosphonate therapy, which showed complete resolution of previously seen calcified lesions. At her next follow up visit, after being on bisphosphonates for 10 months, she no longer had to use Gower's maneuver with standing, and had equal range of motion in her bilateral lower extremities. A repeat CT scan was performed 11 months after therapy, which, similar to the $\mathrm{x}$-ray, showed complete resolution of the previously seen soft tissue calcifications (Figs. 4 and 5). With complete resolution of the calcinosis, her alendronate was discontinued at her next follow-up visit. Throughout the duration of treatment, she did not report any of the short-term side effects such as GI irritation or new bone pain related to her bisphosphonate therapy.

\section{Discussion and conclusion}

Our case demonstrates the successful use of oral bisphosphonates in resolving soft tissue calcinosis in a patient with JDM-SLE overlap syndrome. In the treatment of

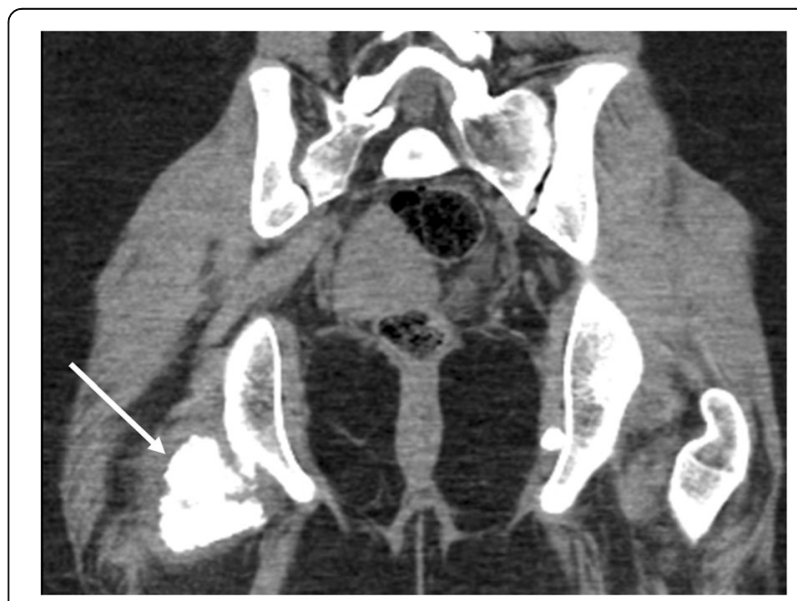

Fig. 2 Coronal CT image of the pelvis from the same examination as Fig. 1 again shows the large area of soft tissue calcification in the right ischiofemoral space (white arrow) 


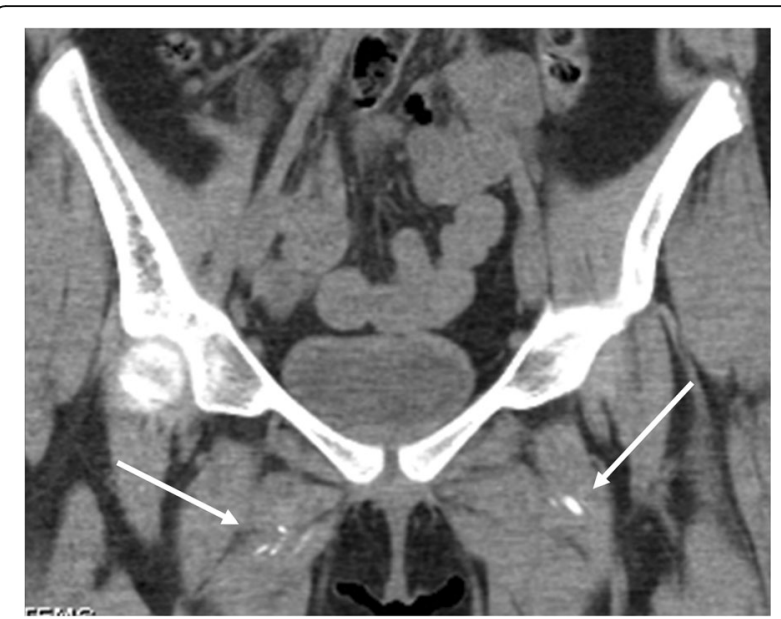

Fig. 3 Coronal CT image from the same examination as Fig. 1 shows additional areas of soft tissue calcification in the right and left adductor musculature (white arrows)

diseases causing excessive bone resorption such as osteoporosis, bisphosphonates work by inhibiting osteoclast function through various different mechanisms. Bisphosphonates have also been shown to inhibit the formation of calcium phosphate salts, thus making them a theoretical treatment option for disorders of excessive calcinosis such as JDM [11]. In previous case studies, it has been reported that bisphosphonates have been used successfully to treat calcinosis in patients with JDM [7, 8]. In one of the largest studies to date, six patients with JDM and calcinosis were all treated with bisphosphonates, and four of the six patients demonstrated complete resolution [7]. Additionally, in a large survey

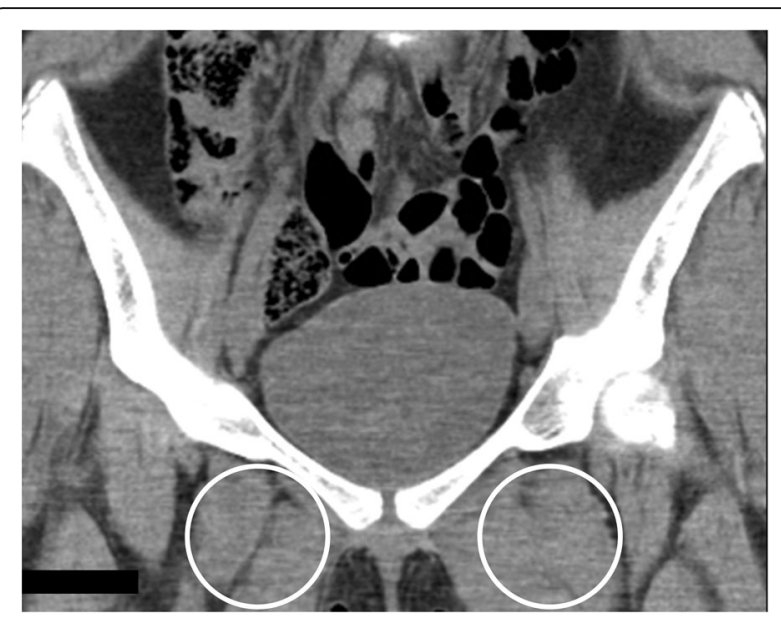

Fig. 5 Coronal CT image from the same follow-up examination as Fig. 3 shows resolution of the areas of calcification in the adductor musculature (while circles

of pediatric rheumatologists, when asked about treating calcinosis in JDM, 73\% said they would use bisphosphonates, and $60 \%$ ranked them as the most effective treatment [6]. Despite these studies, it is still unclear what the best option for treatment is, and there are currently no recommended guidelines for the treatment of calcinosis in JDM or other rheumatologic diseases. Our case provides further evidence that bisphosphonates can be an effective and safe medication used to treat calcinosis in rheumatologic diseases. Furthermore, while bisphosphonates have been reported as effective use in JDM, its use in other disorders is less widely reported. Our case demonstrates that bisphosphonates can be effective

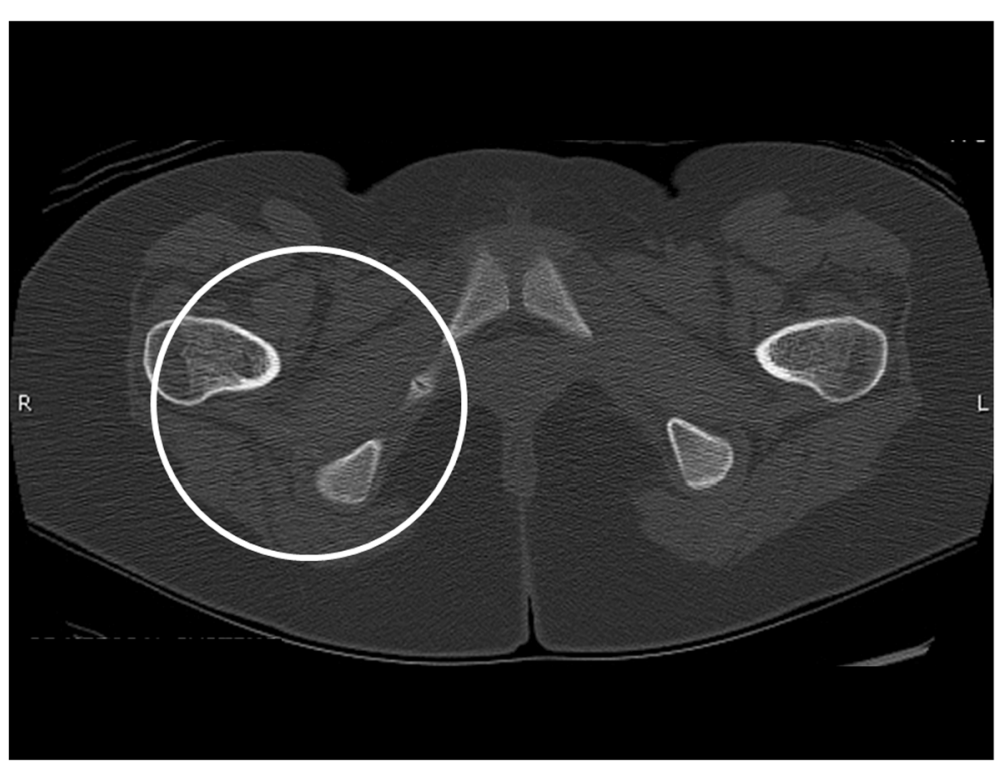

Fig. 4 Axial CT image of the pelvis from a follow-up examination showed complete resolution of the area of bulky calcification (white circle) 
in rheumatologic diseases apart from JDM, in diseases such as overlap syndrome as seen in our patient. While our case report provides important new information, it is limited in the fact that it is a retrospective, observational report on a single patient. It would be beneficial to perform large, case-controlled studies in the future to further evaluate the overall effectiveness and safety of using bisphosphonates in the treatment of pediatric rheumatologic disorders.

\section{Abbreviations}

JDM: Juvenile dermatomyositis; SLE: Systemic lupus erythematosus; MCTD: Mixed connective tissue disorder; ACTD: Autoimmune connective tissue disorder; CMAS: Childhood myositis associated score; IVIG: Intravenous immunoglobulin; AST: Aspartate aminotransferase; ALT: Alanine aminotransferase; LDH: Lactate dehydrogenase; MRI: Magnetic resonance imaging; ANA: Antinuclear antibody; dsDNA: Double stranded DNA; RNP: Ribonucleoprotein; IV: Intravenous; CT: Computed tomography

\section{Acknowledgements}

The authors would like to acknowledge the invaluable work of RP's partner Dr. Deepika Singh, another pediatric rheumatologist, who helped provide our patient with such excellent care that ultimately resulted in the resolution of her symptoms.

\section{Authors' contributions}

RP - primary rheumatologist for our patient, revised and edited the report. MP - performed the literature review and chart review of our patient, and was the primary author in writing the case report. BP - initially interpreted the images for our patient, and formatted the images and wrote the figure keys for this paper. All authors have read and approved the manuscript.

\section{Authors' information}

MP is a third year pediatric resident at Valley Children's Hospital in Madera, California. He completed his osteopathic degree at Touro University in Vallejo, California.

BP is a Pediatric Radiologist at Valley Children's Hospital in Madera, California. $\mathrm{RP}$ is a Pediatric Rheumatologist at Valley Children's Hospital in Madera, California. She is a core faculty member and Clinical Assistant Professor for Valley Children's Pediatric Residency program affiliated with Stanford University School of Medicine.

\section{Funding}

This case report did not receive any specific grant from funding agencies in the public, commercial or non-profit sectors. All authors read and approved the final manuscript.

\section{Availability of data and materials}

Data sharing is not applicable to this article as no datasets were generated or analyzed during the current study.

\section{Ethics approval and consent to participate}

Written and verbal consent obtained from patient and parent (legal guardian).

\section{Consent for publication}

Written consent to publish the above information, including all images, was obtained from the patient's parent, who is her legal guardian.

\section{Competing interests}

The authors declare that they have no competing interests.

Received: 11 September 2020 Accepted: 20 January 2021

Published online: 26 February 2021

\section{References}

1. Mathiesen P, Hegaard H, Herlin T, Zak M, Pedersen FK, Nielsen S. Long-term outcome in patients with juvenile dermatomyositis: a cross-sectional follow- up study. Scand J Rheumatol. 2012. https://doi.org/10.3109/03009742.2011. 608376.

2. Clemente G, Piotto DG, Barbosa C, Peracchi OA, Len CA, Hilario MO, et al. High frequency of calcinosis in juvenile dermatomyositis: a risk factor study. Rev Bras Rheumatol. 2012;52(4):549-53.

3. Gara S, Jamil RT, Litaiem N. Juvenile dermatomyositis. StatPearls StatPearls Publishing https://www.ncbi.nlm.nih.gov/books/NBK534236. Accessed 31 Mar 2020.

4. Balin SJ, Wetter DA, Andersen LK, Davis MDP. Calcinosis cutis occurring in association with autoimmune connective tissue disease: the Mayo Clinic experience with 78 patients, 1996-2009. Arch Dermatol. 2012. https://doi. org/10.1001/archdermatol.2011.2052.

5. Miyamae T, Mori M, Inamo Y, Kohno Y, Takei S, Maeda M, et al. Multi-center analysis of calcinosis in children with juvenile dermatomyositis. Ryumachi. 2003;43(3):538-43.

6. Orandi AB, Baszis KW, Dharnidharka VR, Huber AM, Hoeltzel MF; CARRA Juvenile Myositis subgroup. Assessment, classification and treatment of calcinosis as a complication of juvenile dermatomyositis: a survey of pediatric rheumatologists by the childhood arthritis and rheumatology research alliance (CARRA). Pediatr Rheumatol Online J. 2017; doi:https://doi. org/10.1186/s12969-017-0199-4.

7. Tayfur AC, Topaloglu R, Gulhan B, Bilginer Y. Bisphosphonates in juvenile dermatomyositis with dystrophic calcinosis. Mod Rheumatol. 2015. https:// doi.org/10.3109/14397595.2014.988197.

8. Martillotti J, Moote D, Zemel L. Improvement of calcinosis using pamidronate in a patient with juvenile dermatomyositis. Pediatr Radiol. 2014. https://doi.org/10.1007/s00247-013-2738-6.

9. Batch JA, Couper JJ, Rodda C, Cowell CT, Zacharin M. Use of bisphosphonate therapy for osteoporosis in childhood and adolescence. J Pediatr Child Health. 2003;39(2):88-92.

10. Huber AM, Giannini EH, Bowyer SL, et al. Protocols for the initial treatment of moderately severe juvenile dermatomyositis: results of a Children's arthritis and rheumatology research Alliance consensus conference. Arthritis Care Res (Hoboken). 2010;62(2):219-25. https://doi.org/10.1002/acr.20071.

11. Fleisch H. Bisphosphonates: mechanisms of action. Endocr Rev. 1998;19(1): $80-100$.

\section{Publisher's Note}

Springer Nature remains neutral with regard to jurisdictional claims in published maps and institutional affiliations.
Ready to submit your research? Choose BMC and benefit from:
- fast, convenient online submission
- thorough peer review by experienced researchers in your field
- rapid publication on acceptance
- support for research data, including large and complex data types
- gold Open Access which fosters wider collaboration and increased citations
- maximum visibility for your research: over $100 \mathrm{M}$ website views per year
At BMC, research is always in progress.
Learn more biomedcentral.com/submissions 